\title{
3D TRUS Guided Robot Assisted Prostate Brachytherapy
}

\author{
Zhouping Wei, Mingyue Ding, Donal Downey, and Aaron Fenster \\ Imaging Research Laboratories, Robarts Research Institute, \\ 100 Perth Drive, London, ON N6A 5K8, Canada \\ \{zwei, mding, ddowney, afenster\}@imaging.robarts.ca
}

\begin{abstract}
This paper describes a system for dynamic intraoperative prostate brachytherapy using 3D ultrasound guidance with robot assistance. The system consists of 3D transrectal ultrasound (TRUS) imaging, a robot and software for prostate segmentation, 3D dose planning, oblique needle segmentation and tracking, seed segmentation, and dynamic re-planning and verification. The needle targeting accuracy of the system was $0.79 \mathrm{~mm} \pm 0.32 \mathrm{~mm}$ in a phantom study.
\end{abstract}

\section{Introduction}

Transperineal prostate brachytherapy provides an improved alternative for minimalinvasive treatment of prostate cancer [1]. Current prostate brachytherapy involves placing about 80 to 100 radioactive seeds (e.g. ${ }^{125} \mathrm{I}$ or ${ }^{103} \mathrm{Pd}$ ) into the prostate based on a predetermined plan (pre-plan). A template with a rectilinear pattern of holes is used to guide the implantation needles, loaded with radioactive seeds, to be inserted into the prostate according to the pre-plan. The needle insertion into the prostate is carried out under two-dimensional (2D) transrectal ultrasound (TRUS) guidance [2]. With accurate placement of the seeds, the high dose of radiation is expected to be confined, essentially, to the prostate, dramatically limiting treatment-related complications by minimizing radiation to nearby organs, such as the bladder and rectum.

However, it has been widely recognized that current prostate brachytherapy is still susceptible to variability. A dynamic intraoperative procedure, in which all steps are performed in one session, including planning, monitoring of prostate changes, dynamic re-planning, optimal needle insertion including oblique trajectories and automatic seed localization in US images, will help to solve some of the problems with current prostate brachytherapy [3].

To achieve dynamic intraoperative prostate brachytherapy, we developed a 3D TRUS guided and robot assisted system with new software. In this paper, we describe the development of the system and the related algorithms, and report on the targeting accuracy and variability achievable with the system.

\section{Methods}

\subsection{System Description}

Our prototype system (see Fig. 1) consists of a commercial robot, and a 3D TRUS imaging system including a B\&K 2102 Hawk US system (B\&K, Denmark) with a sidefiring $7.5 \mathrm{MHz}$ TRUS transducer coupled to a rotational mover for 3D imaging [4]. The 
mover rotates the transducer about its long axis, while 2D US images are digitized and reconstructed into a 3D image while the images are acquired. A one-hole needle guide is attached to the robot arm, so that the position and orientation of the needle targeting can be changed as the robot moves. The robot and 3D TRUS coordinate systems are integrated with robot and image calibrations of the coordinate systems [5]. As a result, the robot can be controlled to target any point in 3D TRUS images, along any trajectory including oblique to avoid pubic arch

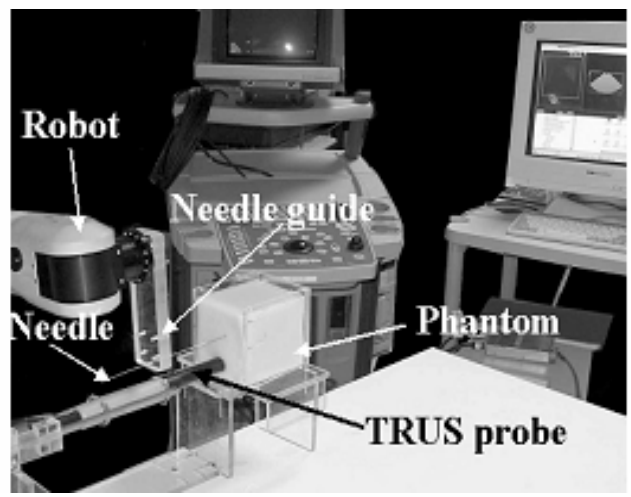

Fig. 1. 3D TRUS guided and robotic assisted prostate brachytherapy system interference (PAI).

The software tools included in the system provide following functions:

1. Semiautomatic prostate segmentation in 3D TRUS images: We used the Discrete Dynamic Contour (DDC) method for semi-automatic prostate segmentation in 3D TRUS images [6]. First, the 3D TRUS image of the prostate is re-sampled into slices using a rotational re-slicing method (Fig. 2(a)). Then, in an initial slice, four or more points are chosen on the boundary of the prostate to obtain an initial contour using a cardinal-spline (Fig. 2(b)). In the next step, the initial contour is refined using the DDC method to obtain a prostate-fitted contour in the initial slice (Fig. 2(c)). The refined prostate contour in the initial slice is then propagated to adjacent slices and refined until the prostate boundaries in all slices have been segmented.
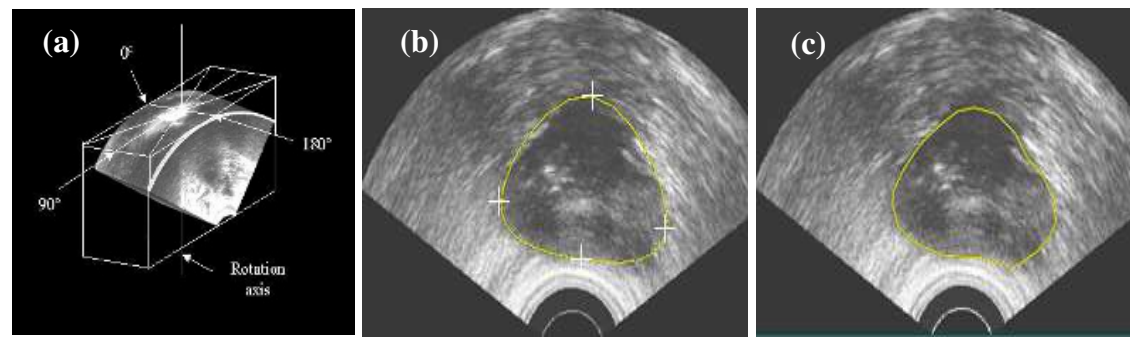

Fig. 2. Prostate segmentation. (a) Rotational re-slicing. (b) Initial slicing. (c) Refine the contour.

2. 3D prostate dose planning: Real time dose calculation is performed using TG43 formalism. Geometric optimization followed by simulated annealing is used to obtain the optimal dose distribution for pre-planning. The dose distribution is evaluated using dose volume histograms (DVH) for the delineated organs, as well as for the implant volume. The plan can be performed and displayed in 3D volume view, orthogonal planes view, transverse view, and needle rendered view. The user can switch on and off the contours, isodose curves, needles, etc., in order to view each separately, and obtain the dose at any point by simply clicking the mouse on the transverse image or 
by typing the coordinates. Dose volume histograms for different plans from the same patient or different DVH from the same plan can also be displayed for comparison.

3. Oblique needle segmentation and tracking: The aim of oblique needle segmentation is to determine needle's position and orientation, so that rapid dynamic replanning could be performed, based on the actual needle trajectory and seed locations to obtain an optimum 3D dose distribution within the prostate. The oblique needle segmentation algorithm includes six steps [7]: 1) pre-scan to obtain the image prior to the insertion of the needle; 2) live-scans to obtain the images as the needle is being inserted; 3) image subtraction to obtain a difference images between the pre-scan and live-scans; 4) thresholding to remove background noise (Fig. 3(a)); 5) further removal of spurious needle voxels to obtain the needle candidate voxels (Fig. 3(b)); 6) linear regression of needle candidate voxels to obtain the needle vector in 3D TRUS image coordinate system (Fig. 3(c)).

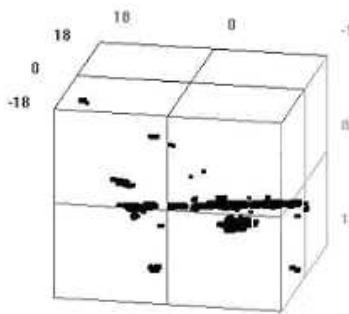

(a)

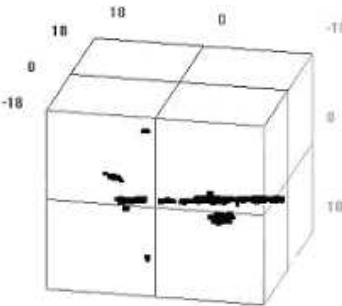

(b)

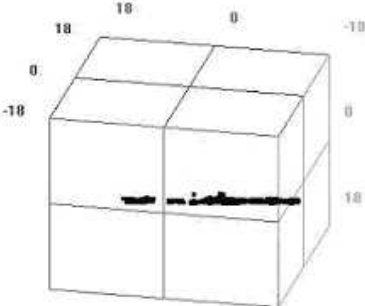

(c)

Fig. 3. Oblique needle segmentation procedure. (a) 3D difference map after the thresholding processing; (b) 3D difference map after the spurious needle voxels have been removed; (c) 3D difference map ready for final linear regression. Black regions are the needle candidate clusters.

4. Automatic seed localization in 3D TRUS images: Automated seed localization is important for intraoperative evaluation of dose delivery, which permits the identification of under-dosed regions, need for remedial seed placement, and ensures that the entire prostate receives the prescribed dose. The automatic seed segmentation algorithm is composed of following five steps: 1) 3D needle segmentation to obtain the needle position when implanting the seeds; 2) reducing the search space by volume cropping along the detected needle, as the implanted seeds are close to the needle; 3 ) non-seed structure removal based on model of orthogonal projections of the needle; 4) seed candidate recognition using 3D line segment detection; and 5) localization of seed positions using a peak detection algorithm described in [8] to localize the center of the seeds.

\subsection{System Evaluation}

To assess the performance of the 3D TRUS guided and robotic assisted system, we used tissue-mimicking prostate phantoms made from agar and contained in a Plexiglas box. A hole in the side allowed insertion of the TRUS transducer into the phantom, simulating the rectum (Fig. 4). Each phantom contained of two rows of $0.8 \mathrm{~mm}$ diameter stainless steel beads. The bead configurations formed a $4 \times 4 \times 4 \mathrm{~cm}$ polyhedron to simulate the approximate size of a prostate. These beads were scanned using 
the 3D TRUS system, and the positions of these seeds were determined manually in 3D TRUS image coordinate system. Then, these positions were transferred into the robot coordinate system, and the robot was controlled to guide the needle to target these beads. The displacements between the preinsertion bead position and the needle tip after the needle has been inserted into the phantom were used to analyze the needle insertion accuracy. A 3D principal component analysis (PCA) was performed to analyze needle targeting accuracy. The elements in the covariance matrix used for PCA analysis are defined as:

$S_{i j}=\frac{\sum_{k=1}^{8}\left(x_{i k}-\bar{x}_{i}\right)\left(x_{j k}-\bar{x}_{j}\right)}{(n-1)} \quad(i, j=1,2,3)$

(1) Fig. 4. The two different prostate phantom types with four different bead configurations were used for the needle targeting accuracy experiment. The needle entered from left-hand side, parallel to the $\mathrm{x}$-axis, in each case.

where, $x_{i k}$ is the displacement between the middle of the pre-insertion bead and the needle tip. $i$ and $j$ represent the direction component (i.e., $x, y$ or $z), k$ is the target index $(k=1,2, \ldots, 8$, i.e., for
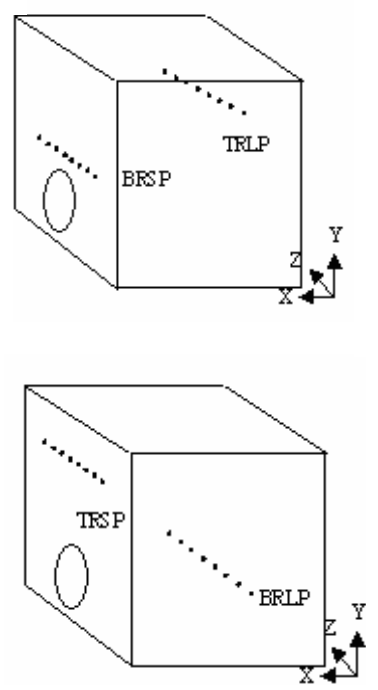
each bead, 8 targeting trials were performed) in

each of four bead configurations as shown in Fig. 4. $\bar{x}_{i}=\frac{1}{8} \sum_{k=1}^{8} x_{i k}$ is the mean displacement in the $i$ th component, and $n=8$. Solving the determinant equation:

$$
|\boldsymbol{S}-\lambda \boldsymbol{I}|=0
$$

where $\boldsymbol{I}$ is the identity matrix. $\boldsymbol{S}$ is the covariance matrix, whose elements have been determined using Eq. (1). Eqs. (1) and (2) produce a third degree polynomial equation, with roots that are the eigenvalues of $S, \lambda_{i}(i=1,2,3)$, and the corresponding normalized eigenvectors $\boldsymbol{u}_{\mathrm{i}}(i=1,2,3)$. The eigenvalues give us the variance along the corresponding eigenvectors, with maximum variance occurring along the principal axis. The two other axes, orthogonal to the first and each other, account for the remaining variance, which is calculated as the corresponding eigenvalues of the covariance matrix. The principal components, i.e., the orthonormal eigenvectors, $\mathrm{u}_{i}$, are not necessarily parallel to the image coordinate axes.

Three-dimensional 95\% confidence intervals were calculated for the eight bead locations for each bead configuration. We assumed that the needle targeted the origin of the coordinate system, and plotted the $95 \%$ confidence interval as an ellipsoid about the average needle position. The orientation and length of the ellipsoid axis were given by the eigenvectors and eigenvalues from the PCA. Because the variance is the square of the STD, the length of the $95 \%$ confidence interval in one direction along each of the principal axes, $\boldsymbol{u}_{\mathrm{i}}(i=1,2,3)$, is given by: 


$$
a_{i}=2 \sqrt{\lambda_{i}} \quad(i=1,2,3)
$$

where $\lambda_{i}$ is the eigenvalue of the covariance matrix. The equation of the resulting ellipsoid is then given by:

$$
\sum_{i} \frac{\left(u_{i}\right)^{2}}{a_{i}^{2}}=1 \quad(i=1,2,3)
$$

The ellipsoid equation was transformed back to the image coordinate system, and translated so that its center was at the average needle position relative to the preinsertion bead position. The displacements of the measured positions of the needle tip relative to the preinsertion bead positions at the origin were plotted for all eight targeting attempts for each bead configuration along with the $95 \%$ ellipsoid intervals. The volume of $95 \%$ ellipsoid was calculated by:

$$
\mathrm{V}=\frac{4 \pi}{3} \prod_{i=1}^{3} a_{i}
$$

$a_{i}(i=1,2,3)$ are the ellipsoid axis lengths determined by Eq. (3). Projections through the ellipsoid and needle data were also plotted.

To assess the performance of the prostate segmentation algorithm, we compared the algorithm segmented prostate with manual segmentation. Due to the robot's high positioning and angulation accuracy, we used the robot as a "gold standard" to assess the performance of the needle segmentation algorithm. We compared the results of algorithm segmentation to the values measured by the robot.

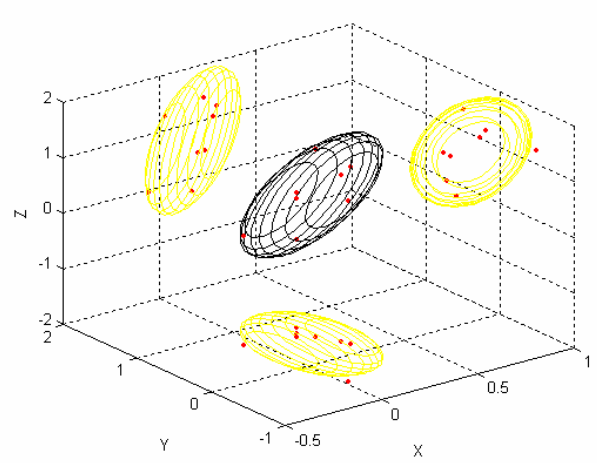

Fig. 5. An ellipsoid representing $95 \%$ confidence interval for needle targeting. The target locates at the origin of the coordinate system. The small dots are the real needle tip positions.

\section{Results}

Evaluation of the system showed that needle can be used to target positions in agar phantoms with a mean error of $0.79 \mathrm{~mm} \pm 0.32 \mathrm{~mm}$. The 3D 95\% confidence ellipsoids (see Fig. 5) were found to have total volumes ranging from $0.54 \mathrm{~mm}^{3}$ to $1.92 \mathrm{~mm}^{3}$, depending on the location of the targets with respect to the ultrasound probe and insertion distance. The size of the ellipsoid, representing the $95 \%$ confidence interval, describes the random errors of the needle targeting. The random error results from poorer resolution in the 3D scanning direction $\left(Z_{i}\right)$ due to insufficient sampling of $2 \mathrm{D}$ images and the poorer elevational resolution of the transducer. This image "blurring" 
would be exacerbated for the points farther away from the TRUS transducer, resulting the larger volumes for the targeting of the beads in the top row. Table 1 lists the widths of the $95 \%$ confidence intervals along the primary, secondary and tertiary axis for four different bead configurations.

The distance between the intersection of a radial line with the algorithm segmented surface, and the intersection of the same radial line with the manually outlined surface, was used as the measure of the algorithm's performance. Error analysis showed that the average difference between manual and the prostate segmentation algorithm boundaries was $-0.20 \pm 0.28 \mathrm{~mm}$, the average absolute difference was $1.19 \pm 0.14 \mathrm{~mm}$, the average maximum difference was $7.01 \pm 1.04 \mathrm{~mm}$, and the average volume difference was $7.16 \% \pm 3.45 \%$. Figure 6 is an example of a segmented prostate in a 3D TRUS image. Figure 7 is an example of the 3D prostate dose planning tool showing a surface rendered view of a pre-plan with delineated organs and oblique needle targeting.

The error for the needle segmentation algorithm in determining the needle tip position was $0.8 \mathrm{~mm}$ when the insertion distance was greater than $15 \mathrm{~mm}$. The mean error in determining the needle orientation in the yaw and pitch orientations was $0.20^{\circ}$ and $0.03^{\circ}$ respectively. Figure 8 shows the result of the needle segmentation algorithm in a patient image obtained during a prostate cryotherapy procedure.

The true-positive rate for the seed segmentation algorithm was $100 \%$ for agar and 93\% for chicken phantoms. This is acceptable, because according to Lindsay et al [9], variation in D90 (the lower bound on the minimum dose delivered to any

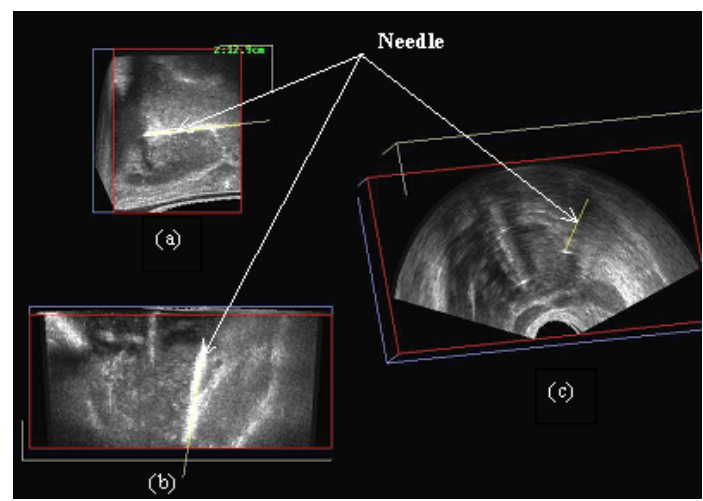

Fig. 8. Segmented needle. (a) Oblique sagittal view; (b) oblique coronal plane; (c) transverse view with a graphical 3D display of the needle targeting superposed on the view. 
$90 \%$ of the target volume) due to inability to localize $5 \%$ of the seeds is negligible. The average distance to the manually segmented seeds was $1.0 \mathrm{~mm}$ for agar and $1.7 \mathrm{~mm}$ for chicken phantoms. The preliminary result for the segmentation time on a PC computer with dual AMD Athlon $1.8 \mathrm{GHz}$ processor was 280 seconds for 14 seeds. This is too long for routine brachytherapy, and we are improving the seed segmentation method so that the segmentation time is limited to less than 1 minute. Figure 9 is an example of the result of the seed segmentation algorithm for localization of the seeds in the 3D TRUS image.

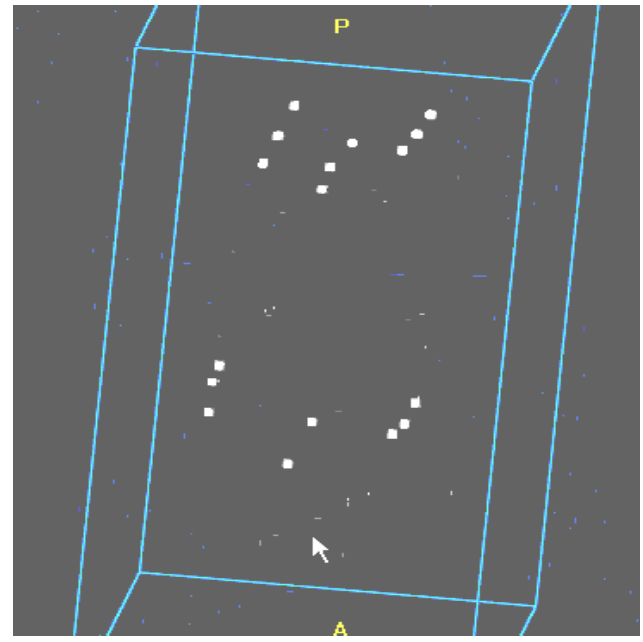

Fig. 9. Localized seeds in 3D TRUS image

\section{Conclusion}

Nath et al. has pointed out that the maximum difference between the needle tip and its planned target allowed in prostate brachytherapy is $5 \mathrm{~mm}$ [10]. Compared with our results from the phantom study, we concluded that with robotic assistance, the brachytherapy needle could be guided to accurately and consistently target any point identified in the 3D TRUS image along various trajectories including oblique. In addition, the software developed in this project provides various functions such as oblique needle segmentation and tracking, 3D dose planning, radioactive seed segmentation in 3D TRUS images, and dynamic re-planning, which are important for an intraoperative procedure. In the future, we will test our system in vivo, which will include realistic tissue deformation and mobility. We expect that the result of this work can provide a tool to achieve dynamic intraoperative prostate brachytherapy using 3D TRUS imaging and robotic assistance together with efficient segmentation software.

Table 1. A description of the $95 \%$ uncertainty intervals for brachytherapy needle insertion

\begin{tabular}{|c|c|c|c|c|c|}
\hline \multirow{2}{*}{ Bead configuration } & \multicolumn{3}{|c|}{$\begin{array}{c}\text { Width of the } 95 \% \text { uncertainty } \\
\text { interval (mm) }\end{array}$} & \multirow{2}{*}{$\begin{array}{l}\text { Center of confi- } \\
\text { dence interval } \\
(\mathbf{m m})\end{array}$} & \multirow{2}{*}{$\begin{array}{c}\text { Ellipsoid } \\
\text { volume } \\
\left(\mathbf{m m}^{3}\right)\end{array}$} \\
\hline & $\begin{array}{l}\text { Primary } \\
\text { axis }\end{array}$ & $\begin{array}{l}\text { Secondary } \\
\text { axis }\end{array}$ & $\begin{array}{l}\text { Tertiary } \\
\text { axis }\end{array}$ & & \\
\hline $\begin{array}{l}\text { Top row, long pene- } \\
\text { tration (TRLP) }\end{array}$ & 1.95 & 1.02 & 0.23 & $(-0.46,0.57,-0.98)$ & 1.92 \\
\hline $\begin{array}{l}\text { Bottom row, short } \\
\text { penetration (BRSP) }\end{array}$ & 1.06 & 0.55 & 0.22 & $(0.38,-0.22,-0.44)$ & 0.54 \\
\hline $\begin{array}{l}\text { Top row, short } \\
\text { penetration (TRSP) }\end{array}$ & 1.77 & 0.94 & 0.24 & $(0.03,0.49,-0.04)$ & 1.68 \\
\hline $\begin{array}{l}\text { Bottom row, long } \\
\text { penetration (BRLP) }\end{array}$ & 1.38 & 0.60 & 0.19 & $(0.17,0.39,0.68)$ & 0.66 \\
\hline
\end{tabular}




\section{References}

1. Blasko J.C., Ragde H., Luse R.W., Sylvester J.E., Cavanagh W., Grimm P.D.: Should brachytherapy be considered a therapeutic option in localized prostate cancer? Urol Clin North Am 23 (1996) 633-650

2. Rifkin M.D.: Ultrasound of the prostate: imaging in the diagnosis and therapy of prostatic disease. Lippincott-Raven Publishers, Philadelphia, New York (1997)

3. Todor D.A., Zaider M., Cohen G.N., Worman M.F., Zelefsky M.J.: Intraoperative dynamic dosimetry for prostate implants. Phys. Med. Biol. 48 (2003) 1153-1171.

4. Fenster A., Downey D.B., Cardinal H.N.: Three-dimensional ultrasound imaging. Phys Med Biol 46 (2001) R67-R99

5. Wei Z., Wan G., Gardi L., Mills G., Downey D.B., Fenster A.: Robot-assisted 3D-TRUS guided prostate brachytherapy: System integration and validation. Med Phys, 31 (2004), 539-548

6. Ding M., Chen C., Wang Y., Gyacskov I., Fenster A.: Prostate segmentation in 3D US images using the cardinal-spline-based discrete dynamic contour. In: Proc. SPIE Medical Imaging 2003 Vol. 5029: Visualization, Image-guided procedures, and display, San Diego, CA, USA, February 16-18, 2003, pp. 69-76

7. Wei Z., Gardi L., Downey D.B., Fenster A.: Oblique needle segmentation for 3D TRUSguided robot aided transperineal prostate brachytherapy. In: Proc. IEEE International Symposium in Biomedical Imaging (ISBI'04), Arlington, VA, USA, April 15-18, 2004

8. Ding M., Wei Z., Downey D.B., Fenster A.: Automated seed localization for intraoperative prostate brachytherapy based on $3 D$ line segment patterns. In: Proc. SPIE Medical Imaging 2005 Vol. 5744: Visualization, Image-guided procedures, and display, San Diego, CA, USA, February 13-15, 2005, pp. 417-424

9. Lindsay P.E., Van Dyk J., Battista, J.J: A systematic study of imaging uncertainties and their impact on ${ }^{125}$ I prostate brachytherapy dose evaluation. Med Phys, 30 (2003), 18971907

10. Nath S, Chen Z, Yue N, Trumpore S, Peschel S. Dosimetric effects of needle divergence in prostate seed implant using ${ }^{125} \mathrm{I}$ and ${ }^{103} \mathrm{Pd}$ radioactive seeds. Med Phys, 27 (2000), 10581066 